\title{
OPEN Biological impacts on the lungs in rats internally exposed to radioactive ${ }^{56} \mathrm{MnO}_{2}$ particle
}

\author{
Nariaki Fujimoto ${ }^{1 凶}$, Bakhyt Ruslanova², Zhaslan Abishev², Nailya Chaizhunussova², \\ Dariya Shabdarbayeva ${ }^{2}$, Gaukhar Amantayeva ${ }^{2}$, Rakhimzhanova Farida ${ }^{2}$, \\ Marat Sandybayev ${ }^{3}$, Kasuke Nagano ${ }^{4}$, Kassym Zhumadilov ${ }^{5}$, Andrey Kaprin 6 , Sergey Ivanov 6 , \\ Valeriy Stepanenko ${ }^{6} \&$ Masaharu Hoshi ${ }^{7}$
}

To understand the radiation effects of the atomic bombing of Hiroshima and Nagasaki among the survivors, radiation from neutron-induced radioisotopes in soil should be considered in addition to the initial radiation directly received from the bombs. ${ }^{56} \mathrm{Mn}$, which emits both $\beta$ particles and $\gamma$-rays, is one of the dominant radioisotopes created in soil by neutrons from the bomb. Thus we investigated the biological effects of internal exposure to ${ }^{56} \mathrm{MnO}_{2}$ particle in the lung of male Wistar rats comparing to the effects of external ${ }^{60} \mathrm{Co}-\gamma$ irradiation. Absorbed doses of internal irradiation of lungs were between 25 and $65 \mathrm{mGy}$ in ${ }^{56} \mathrm{MnO}_{2}$-exposed animals, while the whole body doses were between 41 and $100 \mathrm{mGy}$. Animals were examined on days 3 and 61 after the exposure. There were no remarkable pathological changes related to ${ }^{56} \mathrm{MnO}_{2}$ particle exposure. However, mRNA and protein expressions of aquaporin 5 increased significantly in the lung tissue on day 3 postexposure in ${ }^{56} \mathrm{MnO}_{2}$ groups (by 1.6 and 2.9 times, respectively, in the highest dose group). Smad7 mRNA expression was also significantly elevated by $30 \%$ in the highest dose group of ${ }^{56} \mathrm{MnO}_{2}$. Our data demonstrated that internal exposure to ${ }^{56} \mathrm{MnO}_{2}$ induced significant biological responses including gene expression changes in the lungs, while external ${ }^{60} \mathrm{Co}-\gamma$ irradiation of $2 \mathrm{~Gy}$ did not show any changes.

On the atomic bombing in Hiroshima and Nagasaki, Japan, initial radiation directly from the explosions caused major biological effects. However, residual radiation may have played a role because the people who moved to these cities soon after the detonations not received any direct radiation but exposed to residual-radioactive dust were reported to suffer from various acute radiation syndrome ${ }^{1}$. The radioisotopes produced in soil by the neutron from the atomic bomb included ${ }^{24} \mathrm{Na},{ }^{42} \mathrm{~K}$, and ${ }^{56} \mathrm{Mn}^{2}$. Considering human exposures, ${ }^{56} \mathrm{Mn}$, which was existed as insoluble ${ }^{56} \mathrm{MnO}_{2}$ particles, was one of the most important sources of residual radiation. However, there had been no studies examining the effects of internal exposure to ${ }^{56} \mathrm{Mn}$ before. Then we initiated to investigate the biological effects of this residual radioisotope by exposing laboratory rats to ${ }^{56} \mathrm{MnO}_{2}$ particles and reported possible pathological changes in the lung ${ }^{3}$. Although our previous report consisting of two separate exposure experiments pioneered the investigations of the biological effects of ${ }^{56} \mathrm{Mn}$ particles in animals, the obtained biological data were limited. In the first exposure experiment, only one rat was examined at each time point after exposure to ${ }^{56} \mathrm{Mn}$ particles with a single dose of radiation. In the second exposure, the radiation dose did not reach the level as planned, so the pathological results were less clear. Therefore, a new study to investigate the effects of ${ }^{56} \mathrm{Mn}$ on the lung had been awaited to validate our previous findings and to obtain more detailed data (Supplementary Information).

In the present study, we exposed the necessary numbers of male Wistar rats to three different doses of radioactive ${ }^{56} \mathrm{MnO}_{2}$ powder, or stable $\mathrm{MnO}_{2}$ powder to further examine our previous findings. To compare the effects of internal irradiation by ${ }^{56} \mathrm{MnO}_{2}$ with the external irradiation's effects, a group exposing to ${ }^{60} \mathrm{Co}-\gamma$ at 2 Gy, which is substantially higher than the predicted whole body doses by ${ }^{56} \mathrm{MnO}_{2}$, was also prepared. The dosimetry data indicated that the organ distribution of internal radiation in the present study was similar to the previous results $^{4}$, suggesting good reproducibility in the ${ }^{56} \mathrm{MnO}_{2}$ exposure process ${ }^{5}$. In general, thoracic external radiation

\footnotetext{
${ }^{1}$ Research Institute for Radiation Biology and Medicine, Hiroshima University, Hiroshima, Japan. ${ }^{2}$ Semey Medical University, Semey, Kazakhstan. ${ }^{3}$ Center of Nuclear Medicine and Oncology of Semey, Semey, Kazakhstan. ${ }^{4}$ Nagano Toxicologic-Pathology Consulting, Kanagawa, Japan. ${ }^{5}$ L.N. Gumilyov Eurasian National University, Nur-Sultan, Kazakhstan. ${ }^{6}$ National Medical Research Center of Radiology, Ministry of Health of Russian Federation, Obninsk, Russia. ${ }^{7}$ The Center for Peace, Hiroshima University, Hiroshima, Japan. ${ }^{\boxplus}$ email: nfm@ @hiroshima-u.ac.jp
} 


\begin{tabular}{|l|l|l|l|}
\hline Groups & Body weight $(\mathrm{g})$ & Lung $(\mathrm{g})$ & Lung (relative) $(\mathrm{g} / \mathrm{kg}$ bw) \\
\hline Day 3 \\
\hline Control & $248 \pm 16$ & $1.50 \pm 0.15$ & $6.0 \pm 0.5$ \\
\hline Cold Mn & $235 \pm 14$ & $1.46 \pm 0.12$ & $6.3 \pm 0.6$ \\
\hline Mn56x1 & $235 \pm 11$ & $1.29 \pm 0.07$ & $5.6 \pm 0.4$ \\
\hline Mn56x2 & $245 \pm 16$ & $1.52 \pm 0.12$ & $6.3 \pm 0.5$ \\
\hline Mn56x3 & $237 \pm 12$ & $1.35 \pm 0.08$ & $5.7 \pm 0.2$ \\
\hline Co-60 & $234 \pm 14$ & $1.29 \pm 0.09$ & $5.6 \pm 0.4$ \\
\hline Day 61 & \multicolumn{5}{|l}{} \\
\hline Control & $330 \pm 17$ & $2.10 \pm 0.13$ & $6.5 \pm 0.5$ \\
\hline Cold Mn & $337 \pm 19$ & $1.78 \pm 0.05$ & $5.3 \pm 0.3$ \\
\hline Mn56x1 & $371 \pm 21$ & $1.82 \pm 0.13$ & $4.9 \pm 0.2$ \\
\hline Mn56x2 & $337 \pm 17$ & $1.99 \pm 0.13$ & $6.0 \pm 0.5$ \\
\hline Mn56x3 & $353 \pm 17$ & $1.84 \pm 0.11$ & $5.2 \pm 0.3$ \\
\hline Co-60 & $328 \pm 23$ & $2.04 \pm 0.12$ & $6.4 \pm 0.5$ \\
\hline
\end{tabular}

Table 1. Body and lung weights in rats exposed to ${ }^{56} \mathrm{MnO}_{2},{ }^{60} \mathrm{Co} \gamma$-rays, and cold $\mathrm{MnO}_{2}$. Each value shows mean $\pm \operatorname{SEM}(\mathrm{n}=6$ or 7 , each group).

exposure at high doses (> $8 \mathrm{~Gy}$ ) induces radiation pneumonitis which eventually leads to pulmonary fibrosis in laboratory animals as well as in humans ${ }^{6-9}$. Investigations of developing mechanisms of pulmonary fibrosis have suggested the involvement of transforming growth factor- $\beta$ (TGF- $\beta$ ) and the related genes, including the Smad genes ${ }^{10-12}$. Aquaporins (AQPs) are water channel molecules that control water transfer across cellular membranes. In the lung, AQP1 is located in microvascular endothelia, while AQP 4 is in airway epithelia and AQP 5 is in type I alveolar epithelial cells, all of which are involved in the transport of liquid to maintain a normal physiological status of the lung ${ }^{13}$. Expressions of AQPs were altered in the pathological conditions: AQP1 expression was decreased in the lung with acute hypoxic lung injury, while adenovirus infection decreased both AQP1 and AQP4 ${ }^{14}$. Thoracic irradiation could also affect the pulmonary expression of AQP1 and AQP5 in rats ${ }^{15}$. Therefore, to identify the radiation effect on the lung, we determined the lung injury related gene expressions including TGF- $\beta$, Smad2, Smad7, collagen I, elastin, and AQPs 1, 4 and 5, in addition to the histological analysis of the lung. The animals were examined on days 3 and 61 postexposure because our previous study indicated that pathological changes occurred in the lung on day 3 and were recovered by day $60^{3}$.

\section{Results}

Estimated doses of irradiation. The estimated accumulated doses of internal irradiation from ${ }^{56} \mathrm{MnO}_{2}$ in each organ were previously described ${ }^{5}$. The absorbed doses of the internal irradiation of the lungs were $25 \pm 3$ (Mn56x1 group), $48 \pm 9.0$ (Mn56x2 group), and 65 \pm 13 (Mn56x3 group), while the doses of internal irradiation of the whole body were $41 \pm 8,91 \pm 3$, and $100 \pm 10 \mathrm{mGy}$, respectively. The external irradiation dose from ${ }^{60} \mathrm{Co}-\gamma$ exposure was $2.0 \pm 0.08 \mathrm{~Gy}$.

Body and lung weights. Body and lung weights on days 3 and 61 postexposure are summarized in Table 1 . There were no significant differences in lung weight on either day.

Histology of the lung. Representative histology of the lung with hematoxylin and eosin (HE) staining on days 3 and 61 postexposure in Mn56x3 and the control are shown in Fig. 1. There were no remarkable changes in the lungs in any group. The structure of the alveoli was normal with no signs of thickening of the alveolus wall. Figure 2 showed representative Elastica van Gieson (EVG) and Azan stained histological sections in Mn56x3 and control. EVG staining did not indicate any deposition of elastin on day 61 postexposure. There were no signs of an increase in collagen in the alveolus wall in Azan staining either.

Effects on mRNA expression levels of Col-I, elastin, TGF- $\beta$, Smad2, and Smad7. Figure 3 shows the expression levels of radiation-induced fibrosis-related genes, Col-I, Elastin, TGF- $\beta$, Smad2, and Smad7. In the Mn56x3 group, the Smad7 mRNA levels were significantly elevated on postexposure days 3 . There were no changes in mRNA level otherwise.

Effects on mRNA expression levels of AQP1, AQP4, and AQP5. Figure 4 presents the mRNA expression of AQPs 1, 4, and 5 in each group. On postexposure day 3, AQP5 mRNA expression significantly increased in the Mn56 groups, while AQP1 and AQ4 mRNA levels were not affected. There were no changes on postexposure day 61 .

AQP5 protein expression. Figure 5 showed the AQP5 protein expression in the lungs. The western blotting analysis demonstrated that AQP5 expression was significantly increased in the protein levels in both Mn56x2 and Mn56x3 groups on postexposure day 3. 
$56 \mathrm{Mnx} 3$

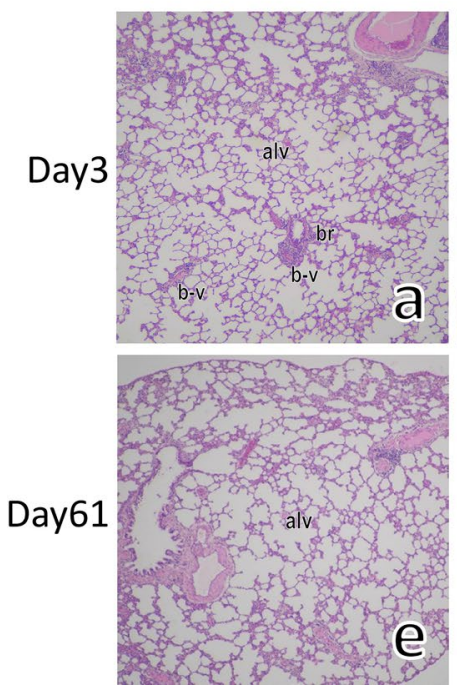

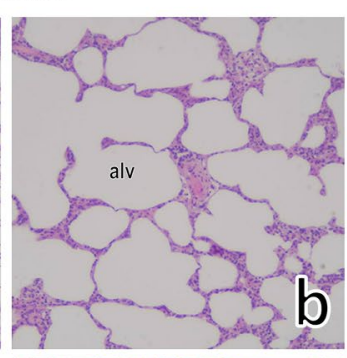

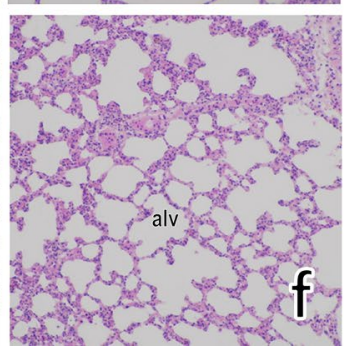

Control
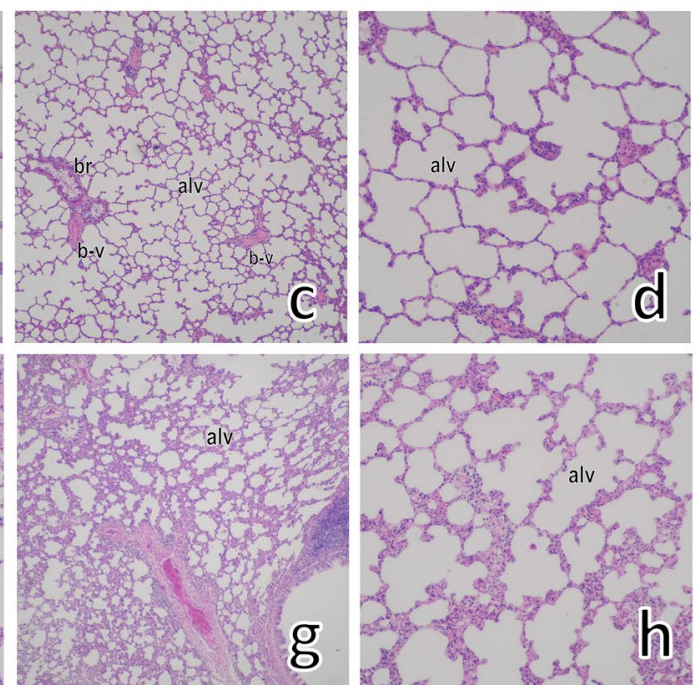

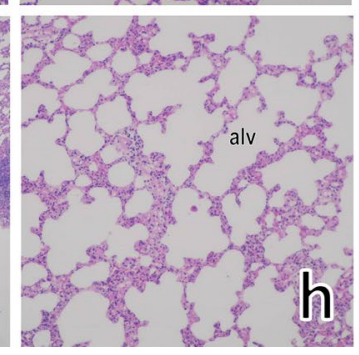

Figure 1. The rats' lungs on days 3 (a-d) and 61 (e-h) after ${ }^{56} \mathrm{MnO}_{2}$ exposure and the control (HE staining). There were no significant histological alternations in the lungs among Mn56x3 (a,b,e,f) and the control (c,d,g,h) groups. The structure of alveoli (alv) was normal with bronchiole (br) and blood vessels (b-v) in the Mn56x3 group. There were no signs of thickening of the alveolus wall even on day 61 postexposure (f) compared to the control (h). Original magnification $\times 40(\mathbf{a}, \mathbf{c}, \mathbf{e}, \mathbf{g})$, or $\times 100(\mathbf{b}, \mathbf{d}, \mathbf{f}, \mathbf{h})$.

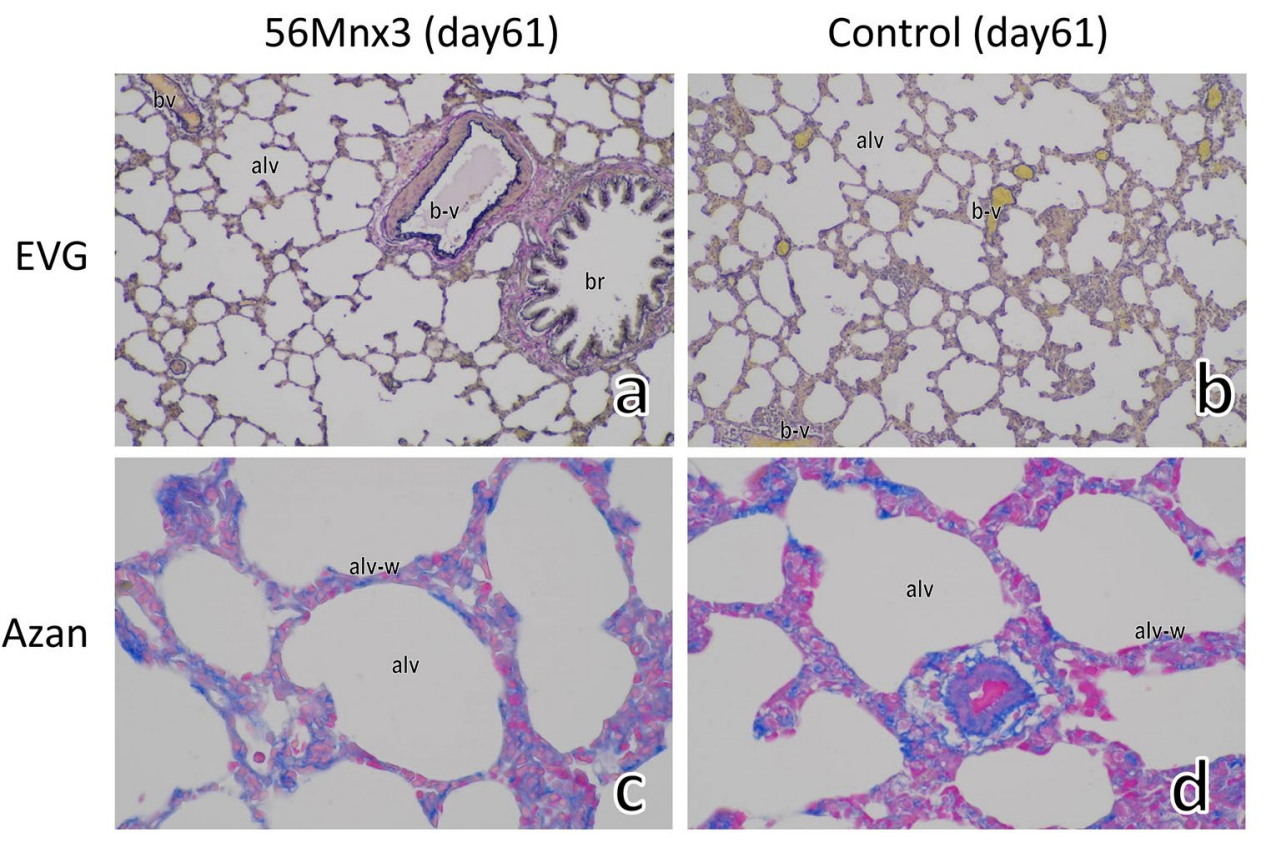

Figure 2. The lung of rats on day 61 after ${ }^{56} \mathrm{MnO}_{2}$ exposure (a,c) and the control (b,d). EVG staining indicated no significant changes in elastin (dark blue or black color) in the alveoli (alv) by ${ }^{56} \mathrm{Mn}$ exposure (a). Azan staining showed no signs of increased collagen deposition (blue color) in the alveolus wall (alv-w) in the ${ }^{56} \mathrm{Mnx} 3$ group (c). Original magnification, $\times 100(\mathbf{a}, \mathbf{b})$, or $\times 400(\mathbf{c}, \mathbf{d})$.

\section{Discussion}

Previously we examined male Wistar rats exposed to ${ }^{56} \mathrm{MnO}_{2}$ powder to understand the biological effects of the possible residual radioactive particles from atomic bombing ${ }^{3}$. Although we reported pathological changes in the lung, it was based on the observation of one animal each day 3,14, or 60 after the exposure. Thus, in the present study, we conducted a new experiment with a substantial number of rats exposed to radioactive ${ }^{56} \mathrm{MnO}_{2}$ particles at three different doses to further examine the effects on the lungs by pathological analysis and determination of the gene and protein expression changes. It was difficult to obtain higher radiation doses of ${ }^{56} \mathrm{Mn}$ than the previous levels due to the technical limitation of our facility. No pathological changes were found even in the Mn56x3 


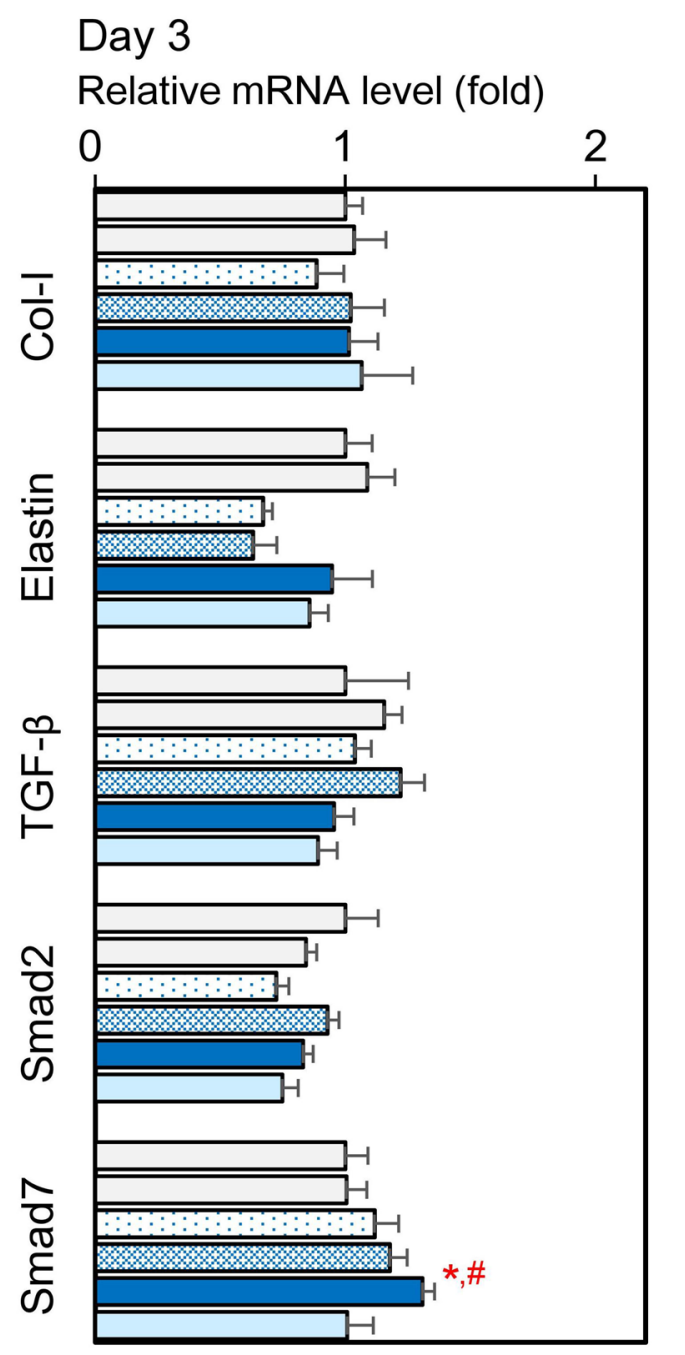

\section{Day 61}

Relative mRNA level (fold)

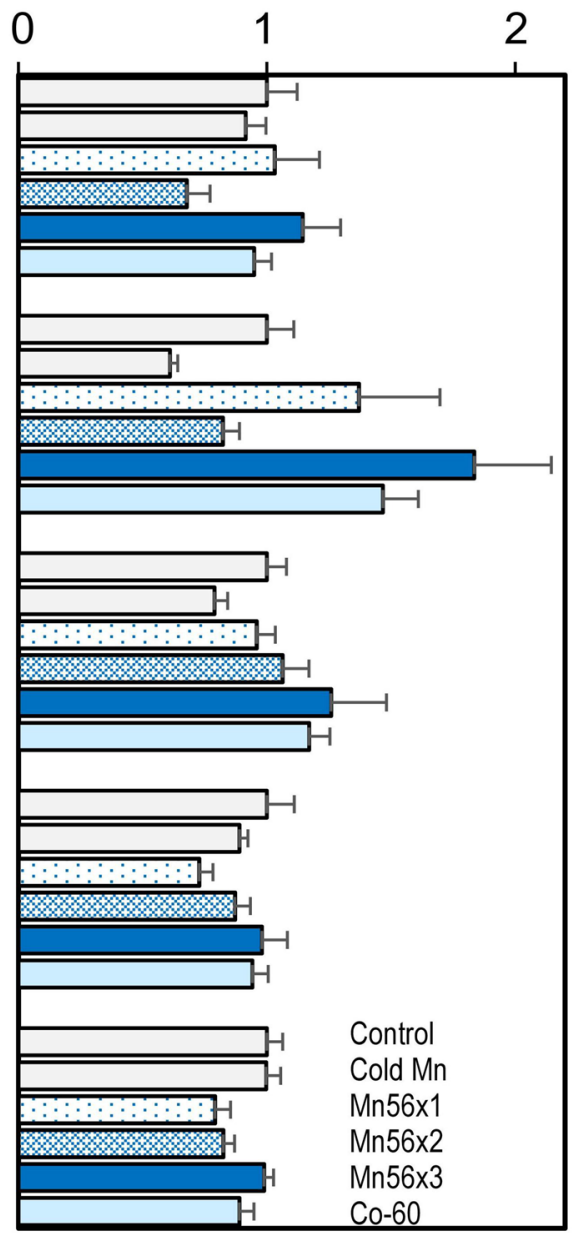

Figure 3. Relative mRNA expression levels of collagen-I, elastin, TGF- $\beta$, Smad 2 and Smad7 genes in the lungs of rats on day 3 (left) and day 61 (right) after the exposure to ${ }^{56} \mathrm{MnO}_{2}$ powder $(\mathrm{Mn} 56 \times 1, \mathrm{Mn} 56 \times 2, \mathrm{Mn} 56 \times 3)$, Cold $\mathrm{MnO}_{2}$ powder (Cold $\mathrm{Mn}$ ) or ${ }^{60} \mathrm{Co}-\gamma$ exposure $(\mathrm{Co}-60)$. ${ }^{*} \mathrm{p}<0.05$ vs. control; ${ }^{\#} \mathrm{p}<0.05$ vs cold $\mathrm{Mn}$.

group. However, the expression in AQP5 increased both mRNA and protein levels on postexposure day 3 in the Mn56x2 and Mn56x3 groups. The Smad7 mRNA expression was also elevated in the Mn56x3 group. However, there were no such changes in the Co-60 group. These data suggest that the internal exposure to ${ }^{56} \mathrm{MnO}_{2}$ powder induced significant biological responses in the lung tissue than external ${ }^{60} \mathrm{Co}-\gamma$ irradiation of 2 Gy although the effects were not pathologically apparent.

It is important to include the effects of residual radiation to understand the radiation effects among the survivors of the atomic bombing of Hiroshima and Nagasaki. Since neutron-activated radioisotopes were present in dust after the bombings, people might have inhaled these radioactive materials and been internally exposed to the radiation. Individuals, who returned early to Hiroshima and Nagasaki after the atomic bombing, were reported to suffer from the symptoms of acute radiation effects ${ }^{1}$. Since there are no data available regarding the absorbed doses in the lung of atomic bomb survivors by these residual radioactive dust, it is not clear whether the radiation doses in the lung found in the present study represent the reality of atomic bomb survivors. However, our data demonstrated the possibility that internal exposure to residual radiation from ${ }^{56} \mathrm{Mn}$ had played a role in developing radiation-induced disorders among the atomic bomb survivors.

High doses of external radiation exposure to the lungs can induce radiation pneumonitis followed by fibrosis in laboratory animals and humans $s^{7,16}$. Radiation-induced lung injury is a major dose-limiting factor in thoracic radiotherapy and has been extensively investigated ${ }^{17}$. TGF- $\beta$ plays an essential role in the development of radiation-induced pulmonary lesions. Serum TGF- $\beta$ could be used as a predictor of radiation pneumonitis in humans ${ }^{18}$. Mice exposed to thoracic irradiation also showed an acute and long-lasting increase in pulmonary TGF- $\beta$ expression ${ }^{19}$. Although TGF- $\beta$ is involved in physiological wound healing and possible subsequent fibrosis, this process often leads to chronic pathological results. TGF- $\beta$ binds to its cell surface serine/threonine kinase receptors, which phosphate the Smad proteins to initiate the transcription of the genes related to diverse biological processes, including fibrosis. Irradiation may change the expression levels of Smad genes, as it was reported 


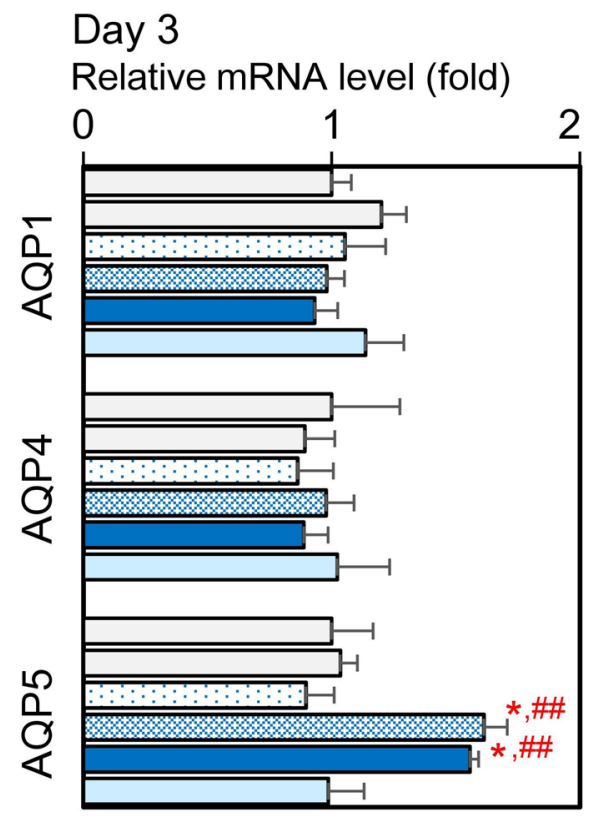

Day 61
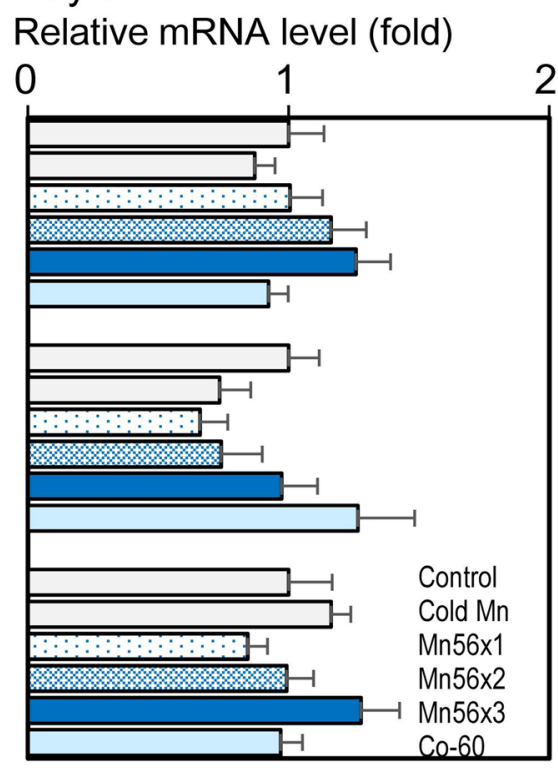

Figure 4. Relative mRNA expression levels of AQP1, AQP4, and AQP5 genes in the lungs of rats on day 3 (left) and day 61 (right) after the exposure to ${ }^{56} \mathrm{MnO}_{2}$ powder (Mn56x1, Mn56x2, Mn56x3), Cold $\mathrm{MnO}_{2}$ powder (Cold $\mathrm{Mn}$ ) or ${ }^{60} \mathrm{Co}-\gamma$ exposure $(\mathrm{Co}-60) .{ }^{*} \mathrm{p}<0.05$ vs. control; ${ }^{\# \#} \mathrm{p}<0.01$ vs cold $\mathrm{Mn}$.

that Smad7 gene expression was increased while Smad2 gene expression was decreased in the lung tissues of
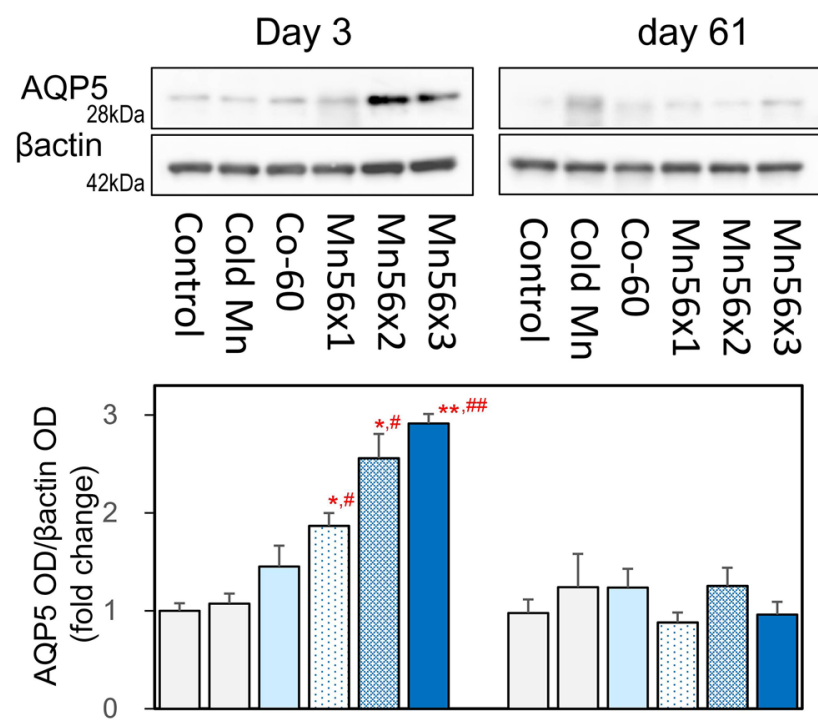

Figure 5. Western blot analysis and densitometry for AQP5 in the lung on postexposure days 3 and 61 . $\beta$-Actin was presented as an internal control. Lysates extracted from the lung were examined. ${ }^{*} \mathrm{p}<0.05,{ }^{* *} \mathrm{p}<0.01$ vs. control; ${ }^{\#} \mathrm{p}<0.05,{ }^{\# \#} \mathrm{p}<0.01$ vs cold $\mathrm{Mn}$.

Wistar rats irradiated with thoracic X-rays at $20 \mathrm{~Gy}^{12}$. In a study with mice exposed to thoracic X-rays at 12 Gy, the Smad signaling was altered on days 2 and 17 postexposure ${ }^{20}$. The present study determined the mRNA levels of TGF- $\beta$, Smad2, and Smad7 genes to examine the possible effects of exposure to ${ }^{56} \mathrm{MnO}_{2}$. External ${ }^{60} \mathrm{Co}$ exposure at $2 \mathrm{~Gy}$ did not cause any changes in these gene expressions. Interestingly, however, Smad7 mRNA levels were significantly increased in the Mn56x3 group on postexposure day 3 , while no differences were noted otherwise. This temporal increase in Smad7 may relate to the healing processes of the lung tissue damages, which were not pathologically apparent. In the development of lung pneumonitis and fibrosis after radiation or chemical exposures, both collagen and elastin contents gradually increase in the lung tissue as early as two weeks after the exposure ${ }^{21,22}$. In the present study, however, there were no significant changes in expressions in either 
collagen or elastin, which suggested the ${ }^{56} \mathrm{Mn}$ exposure may not lead to fibrotic disorders. Aside from ${ }^{56} \mathrm{Mn}$, the effects of radionuclides on the lung have been investigated extensively, although most of these early studies have focused on the development of lung cancer $^{6}$. Some of these studies demonstrated that rats exposed to the a-emitting particles such as ${ }^{239} \mathrm{PuO}_{2}$ or $\beta$-emitting particles including ${ }^{147} \mathrm{Pm}$ indeed induced pneumonitis at the high doses ${ }^{23,24}$. Since our data showed that the change in Smad7 expression occurred even at the low internal dose of $64 \mathrm{mGy}$, it would be interesting to investigate the gene expression profiles in the lung exposure to the other radionuclides as well. Other gene expression changes reported in the irradiated lung representing biological responses were AQPs, a water-selective channel protein family. AQPs are ubiquitously expressed throughout the body and facilitate plasma membrane water permeability and fluid movement ${ }^{25}$. In the lung, AQP1 is expressed in the capillary endothelium and AQPs 4 and 5 are in the epithelium, which plays important roles in the lungs' physiology and pathology ${ }^{26}$. In experimentally induced pulmonary edema in rats, AQP5 expression was found to increase in both mRNA and protein levels, which is probably a physiological response to prevent the lung from edema $^{27}$. For radiation effect studies in rats X-irradiated thoracically, it was reported that AQP5 mRNA level was increased while AQP1 mRNA were decreased in 7 days after the exposures which also suggested a protective role against the radiation-induced inflammation and edema ${ }^{15}$. The present study showed an upregulation of AQP5 mRNA and protein in Mn56x2 and Mn56x3 groups on postexposure day 3, which could be physiological responses and may indicate minor lung injuries caused by ${ }^{56} \mathrm{MnO}_{2}$ exposure, although the histological examination did not indicate any damages. There were no changes in AQP5 or the other gene expression in the Co-60 group, which indicated that internal exposure to ${ }^{56} \mathrm{Mn}$ had higher biological impacts on the lung tissue than the external 2 Gy of $\gamma$-irradiation.

The present study did not identify any pathological effects of ${ }^{56} \mathrm{MnO}_{2}$ exposure on the lung using a significant number of rats. The previous pathological findings were not reproducible. However, it is worth noting that 6-month-old male Wistar rats were used in the previous study, while 10-week-old Wistar rats were used in the present study. The age factor may have caused the discrepancy in the results between the two separate experiments. $\mathrm{Mn}$ is known as a neurotoxic chemical at high doses ${ }^{28}$. In the present study, animals were exposed to $\mathrm{MnO}_{2}$ for only $1 \mathrm{~h}$, which probably did not produce any chemical toxicity of $\mathrm{Mn}$. In fact, there were no significant differences in any parameters examined between cold-Mn group and the control (Table 1, and Figs. 3, $4,5)$. To investigate the radiation-induced lung injuries, mouse models of C57BL and $\mathrm{C} 3 \mathrm{H}$ strains were well established ${ }^{29,30}$. These mouse models would be useful to understand further the effects of ${ }^{56} \mathrm{MnO}_{2}$ particles.

In conclusion, ${ }^{56} \mathrm{MnO}_{2}$ exposure at internal doses of $25-64 \mathrm{mGy}$ did not induce any significant pathological changes in the lungs. However, it caused significant impacts on pulmonary gene and protein expressions, including upregulation of AQP5, and Smad7 expressions, which were not observed in external $\gamma$-irradiation of 2 Gy, indicating very high biological impacts of internal radiation from ${ }^{56} \mathrm{MnO}_{2}$ particles and suggesting the possible roles in radiation effects among atomic bomb survivors.

\section{Methods}

Animals. The animal experiment design was previously described ${ }^{5,31}$. Specific pathogen-free male Wistar rats (10 weeks old) were purchased from the Kazakh Scientific Center of Quarantine and Zoonotic Diseases, Almaty, Kazakhstan. The animals had free access to a basal diet and tap water. The animal facility conditions were maintained as follows: a room temperature of $19-22{ }^{\circ} \mathrm{C}$, a relative humidity of $30-70 \%$, and a 12 -h light cycle. Rats were randomly divided into six groups: Mn56x1 ( $n=17), \operatorname{Mn} 56 x 2(n=17), \operatorname{Mn} 56 \times 3$ ( $n=17)$, Co-60 $(n=14)$, cold $\mathrm{Mn}(n=14)$, and control $(n=14)$. The Mn56x1, Mn56x2, and Mn56x3 groups were exposed to ${ }^{56} \mathrm{MnO}_{2}$ powder $(100 \mathrm{mg})$ at activities of $2.7 \times 10^{8}, 5.5 \times 10^{8}$, and $8 \times 10^{8} \mathrm{~Bq}$, respectively. The cold Mn group was exposed to nonradioactive $\mathrm{MnO}_{2}$ powder $(100 \mathrm{mg})$. Three rats from each Mn56 group were used to determine the absorbed radiation doses. The Co-60 group received 2 Gy of external ${ }^{60} \mathrm{Co} \gamma$-ray whole-body irradiation. From each group, seven rats underwent necropsies on postexposure days 3 and 61 . The rats were sacrificed by removing whole blood under anesthesia with isoflurane (Fujifilm Wako Pure Chemical Co., Tokyo, Japan). The lung was dissected and stored in RNA Save solution (Biological Industries Ltd, Beit Alfa, Israel) for RNA extraction, and part of it was fixed in 10\% formalin. The animal study was approved by the Animal Experiment Ethics Committee of Semey Medical University, Semey, Kazakhstan (document \#3-30.11.2018). The study was carried out in compliance with the ARRIVE guidelines.

Irradiation and dosimetry. Preparation and exposure of ${ }^{56} \mathrm{MnO}_{2}$ powder and the internal dose estimation have been previously described ${ }^{4,5}$. Briefly, $\mathrm{MnO}_{2}$ powder (Rare Metallic Co., Tokyo, Japan; particle diameters range, $1-19 \mu \mathrm{m}$ ) was activated by neutron beam in the Baikal-1 nuclear reactor at the National Nuclear Center, Kurchatov, Kazakhstan. The obtained radioactive ${ }^{56} \mathrm{MnO}_{2}$ powder was sprayed over the rats for $1 \mathrm{~h}$. Then three rats per group were sacrificed for gamma spectrometry and estimated each organ's absorbed dose. All animals were brought to the ${ }^{56} \mathrm{Mn}$ exposure facility. A ${ }^{60}$ Co radiotherapy machine, Teragam K-2 unit (UJP Praha, PrahaZbraslav, Czech Republic) was used (2 Gy at $1.0 \mathrm{~Gy} / \mathrm{min}$ ) for whole-body $\gamma$-ray irradiation. A radiophotoluminescence glass dosimeter, GD-302M (Chiyoda Technol Co., Tokyo, Japan), was used to estimate ${ }^{60}$ Co dose.

Pathology. Formalin-fixed tissues were embedded in paraffin. Sections of $4 \mu \mathrm{m}$ thickness were prepared and stained with HE. Sections were stained with Azan for pulmonary interstitial collagen evaluation. EVG staining was applied for evaluating elastic fiber.

Measurement of mRNA levels by a quantitative reverse transcription-polymerase chain reaction. The details were described previously ${ }^{32}$. Total RNA was prepared using Isogen II (Nippon Gene Co., Tokyo, Japan) from pieces of the lung tissue stored in the RNA Save solution. The cDNA was synthesized by 


\begin{tabular}{|l|l|l|l|}
\hline \multirow{2}{*}{ Gene } & \multirow{2}{*}{ GenBank accession\# } & Q-PCR primer sequences $\left(\mathbf{5}^{\prime} \rightarrow \mathbf{3}^{\prime} \mathbf{)}\right.$ & \\
\cline { 3 - 4 } & Forward & Reverse \\
\hline Aqp1 & NM_012778 & CCACTGGAGAGAAACCAGACG & CTGAGCAGAAGCCCCAGTGT \\
\hline Aqp4 & NM_001317749 & TCTGGACTCAAGCCTTCTGGAA & AGTCCAAAGCAGAGGGAGATGA \\
\hline Aqp5 & NM_012779 & AGGCATCCTGTACTGGCTGG & GAGGAGAAGATGCAGAGGGCT \\
\hline Col1a1 & NM_053304 & AACCTGGATGCCATCAAGGTC & GCTCTCTCCAAACCAGACATGC \\
\hline Ela & BC085910 & TCCTATCTACCCAGGTGGTGG & CACTTTCTCTTCCGGCCACA \\
\hline TGF $\beta$ & AY550025 & GCTGAACCAAGGAGACGGAAT & GAAGGGTCGGTTCATGTCATG \\
\hline Smad2 & AB010147 & GCTCTCCGGCTGAACTGTCT & TGTGACGCATGGAAGGTCTCT \\
\hline Smad7 & NM_030858 & TTGCTGTGAATCTTACGGGAAG & GGTTTGAGAAAATCCATCGGGT \\
\hline
\end{tabular}

Table 2. Q-PCR primers.

incubating $3 \mu \mathrm{g}$ of total RNA + RevaTra Ace $100 \mathrm{U}$ (Toyobo Co., Osaka, Japan) + 20 pmol random hexamers $/ 5$ pmol oligo-dT(15) primers (Takara Bio Inc., Kusatsu, Japan). The quantitative polymerase chain reaction (qPCR) system, StepOnePlus (Applied Biosystems, Life Technologies, Co., Carlsbad, CA, USA), was employed for cDNA measurement with a KAPA SYBR Fast qPCR Kit (Kapa Biosystems, Inc., Woburn, MA, USA). PCR conditions have $30 \mathrm{~s}$ initial denaturation followed by 40 cycles of $5 \mathrm{~s}$ at $95^{\circ} \mathrm{C}$ and $35 \mathrm{~s}$ at $60{ }^{\circ} \mathrm{C}$. Specific primer sets for the lung genes are listed in Table 2 . The PCR products were prepared, and the nucleotide sequences were confirmed by Fasmac Co., Ltd. (Atsugi, Japan) before analysis. The measured mRNA levels were normalized according to the levels of $\beta$-actin mRNA.

Western blot. Lung tissues were homogenized in RIPA lysis buffer containing phosphatase inhibitors (Santa Cruz Biotechnology Inc., Santa Cruz, CA, USA). Two milligrams of each lysate were applied to $12.5 \%$ sodium dodecyl sulfate-polyacrylamide gel electrophoresis and transferred to Hybond-P membranes (GE Healthcare Ltd, Buckinghamshire, UK). After blocking with Blocking One-P (Nacalai Tesque Inc, Kyoto, Japan), the membranes were incubated with anti-AQP5 (GTX11586, x2000, GeneTex, Inc., Irvine, CA, USA) or anti- $\beta$-actin (PM053, 1:1000, MBL Co., Nagoya, Japan). They were washed and incubated with peroxidase-conjugated antirabbit IgG (1:5000, MBL Co.). The protein bands were visualized with Chemi-Lumi reagents (Nacalai Tesque Inc) and image-captured with a CCD camera system, ImageQuant LAS 4000 mini (GE Healthcare Ltd).

Statistical analysis. All values are expressed as mean \pm standard error of the mean. Dunnett's test compared each treated group with the control group or the cold Mn group.

Ethical approval. All methods were carried out in accordance with relevant guidelines and regulations.

Received: 18 January 2021; Accepted: 16 April 2021

Published online: 26 May 2021

\section{References}

1. Imanaka, T., Endo, S., Tanaka, K. \& Shizuma, K. Gamma-ray exposure from neutron-induced radionuclides in soil in Hiroshima and Nagasaki based on DS02 calculations. Radiat. Environ. Biophys. 47, 331-336 (2008).

2. Tanaka, K. et al. Skin dose from neutron-activated soil for early entrants following the A-bomb detonation in Hiroshima: Contribution from $\beta$ and $\gamma$ rays. Radiat. Environ. Biophys. 47, 323-330 (2008).

3. Shichijo, K. et al. Internal exposure to neutron-activated ${ }^{56} \mathrm{Mn}$ dioxide powder in Wistar rats-Part 2: Pathological effects. Radiat. Environ. Biophys. 56, 55-61 (2017).

4. Stepanenko, V. et al. Internal exposure to neutron-activated ${ }^{56} \mathrm{Mn}$ dioxide powder in Wistar rats: Part 1: Dosimetry. Radiat. Environ. Biophys. 56, 47-54 (2017).

5. Stepanenko, V. et al. Internal doses in experimental mice and rats following exposure to neutron-activated ${ }^{56} \mathrm{MnO}_{2}$ powder: Results of an international, multicenter study. Radiat. Environ. Biophys. 59, 683-692 (2020).

6. Coggle, J. E., Lambert, B. E. \& Moores, S. R. Radiation effects in the lung. Environ. Health Perspect. 70, 261-291 (1986).

7. Down, J. D. The nature and relevance of late lung pathology following localized irradiation of the thorax in mice and rats. Br. J. Cancer Suppl. 7, 330-332 (1986).

8. Travis, E. L., Harley, R. A., Fenn, J. O., Klobukowski, C. J. \& Hargrove, H. B. Pathologic changes in the lung following single and multi-fraction irradiation. Int. J. Radiat. Oncol. Biol. Phys. 2, 475-490 (1977).

9. Ward, W. F., Kim, Y. T., Molteni, A., Ts'ao, C. \& Hinz, J. M. Pentoxifylline does not spare acute radiation reactions in rat lung and skin. Radiat. Res. 129, 107-111 (1992).

10. Flanders, K. C. Smad3 as a mediator of the fibrotic response. Int. J. Exp. Pathol. 85, 47-64 (2004).

11. Paun, A., Lemay, A.-M. \& Haston, C. K. Gene expression profiling distinguishes radiation-induced fibrosing alveolitis from alveolitis in mice. Radiat. Res. 173, 512-521 (2010).

12. Xie, L. et al. Integrating microRNA and mRNA expression profiles in response to radiation-induced injury in rat lung. Radiat. Oncol. 9, 1-12 (2014).

13. Verkman, A. S. Role of aquaporins in lung liquid physiology. Respir. Physiol. Neurobiol. 159, 324-330 (2007).

14. Towne, J. E., Harrod, K. S., Krane, C. M. \& Menon, A. G. Decreased expression of aquaporin (AQP) 1 and AQP5 in mouse lung after acute viral infection. Am. J. Respir. Cell Mol. Biol. 22, 34-44 (2000). 
15. Sun, C. Y. et al. The expression of aquaporins 1 and 5 in rat lung after thoracic irradiation. J. Radiat. Res. 55, 683-689 (2014).

16. Marks, L. B. et al. Radiation-induced lung injury. Semin. Radiat. Oncol. 13, 333-345 (2003).

17. Ding, N.-H., Li, J. J. \& Sun, L.-Q. Molecular mechanisms and treatment of radiation-induced lung fibrosis. Curr. Drug Targets 14, $1347-1356$ (2013).

18. Finkelstein, J. N., Johnston, C. J., Baggs, R. \& Rubin, P. Early alterations in extracellular matrix and transforming growth factor $\beta$ gene expression in mouse lung indicative of late radiation fibrosis. Int. J. Radiat. Oncol. Biol. Phys. 28, 621-631 (1994).

19. Rübe, C. E. et al. Dose-dependent induction of transforming growth factor $\beta$ (TGF- $\beta$ ) in the lung tissue of fibrosis-prone mice after thoracic irradiation. Int. J. Radiat. Oncol. Biol. Phys. 47, 1033-1042 (2000).

20. Chen, J. et al. Glycyrrhetinic acid alleviates radiation-induced lung injury in mice. J. Radiat. Res. 58, 41-47 (2017).

21. Bai, Y. H. et al. Collagen gene expression in radiation interstitial pneumonitis. J. Environ. Pathol. Toxicol. Oncol. 13, 231-234 (1994).

22. Venkatesan, N., Punithavathi, D. \& Chandrakasan, G. Biochemical and connective tissue changes in cyclophosphamide-induced lung fibrosis in rats. Biochem. Pharmacol. 56, 895-904 (1998).

23. Dagle, G. E. \& Sanders, C. L. Radionuclide injury to the lung. Environ. Health Perspect. 55, 129-137 (1984).

24. Jackson, I. L., Vujaskovic, Z. \& Down, J. D. A further comparison of pathologies after thoracic irradiation among different mouse strains: Finding the best preclinical model for evaluating therapies directed against radiation-induced lung damage. Radiat. Res. 175, 510-518 (2011)

25. King, L. S. \& Agre, P. Pathophysiology of the aquaporin water channels. Annu. Rev. Physiol. 58, 619-648 (1996).

26. Song, Y. et al. Role of aquaporins in alveolar fluid clearance in neonatal and adult lung, and in oedema formation following acute lung injury: Studies in transgenic aquaporin null mice. J. Physiol. 525, 771-779 (2000).

27. Fabregat, G. et al. Increased expression of AQP 1 and AQP 5 in rat lungs ventilated with low tidal volume is time dependent. PLoS One 9, e114247-e114247 (2014).

28. O’Neal, S. L. \& Zheng, W. Manganese toxicity upon overexposure: A decade in review. Curr. Environ. Health Rep. 2, 315-328 (2015).

29. Scott, B. R. et al. Predicted and observed early effects of combined alpha and beta lung irradiation. Health Phys. 59, 791-805 (1990).

30. Siemann, D. W., Hill, R. P. \& Penney, D. P. Early and late pulmonary toxicity in mice evaluated 180 and 420 days following localized lung irradiation. Radiat. Res. 89, 396-407 (1982).

31. Fujimoto, N. et al. Low-dose radiation exposure with ${ }^{56} \mathrm{MnO}_{2}$ powder changes gene expressions in the testes and the prostate in rats. Int. J. Mol. Sci. 21, 1-10 (2020).

32. Fujimoto, N., Suzuki, T., Ohta, S. \& Kitamura, S. Identification of rat prostatic secreted proteins using mass spectrometric analysis and androgen-dependent mRNA expression. J. Androl. 30, 669-678 (2009).

\section{Acknowledgements}

The authors thank Drs. Almas Azhimkhanov, Vyacheslav Gnyrya, and Alexander Kolbayenkov of the National Nuclear Center of the Republic of Kazakhstan in Kurchatov for their excellent technical help exposing animals to ${ }^{56} \mathrm{MnO}_{2}$ powder. The authors appreciate Drs. Dastan Erzhanov and Oryngazy Aliyev at Semey Medical University for their expert technical assistance of animal necropsies. The authors thank Dr. Hitoshi Sato for his excellent help monitoring external radiation doses. This work was supported by Semey Medical University, Kazakhstan, JSPS Open Partnership Joint Research Projects Grants (H30-31), and JSPS Kakenhi Grants (\#19H01149 and 19KK0266).

\section{Author contributions}

M.H., V.S., N.F. conceived and designed the study, N.F., B.R, Z.A.,G.A., M.S. conducted irradiation, animal studies and gene expression analysis, K.N., B.R., N.F. analyzed the histology, K.Z., A.K., S.I., V.S. conducted dosimetry of irradiation, N.F. drafted the manuscript. All authors have read and approved the final manuscript.

\section{Competing interests}

The authors declare no competing interests.

\section{Additional information}

Supplementary Information The online version contains supplementary material available at https://doi.org/ 10.1038/s41598-021-90443-9.

Correspondence and requests for materials should be addressed to N.F.

Reprints and permissions information is available at www.nature.com/reprints.

Publisher's note Springer Nature remains neutral with regard to jurisdictional claims in published maps and institutional affiliations.

(c) (i) Open Access This article is licensed under a Creative Commons Attribution 4.0 International License, which permits use, sharing, adaptation, distribution and reproduction in any medium or format, as long as you give appropriate credit to the original author(s) and the source, provide a link to the Creative Commons licence, and indicate if changes were made. The images or other third party material in this article are included in the article's Creative Commons licence, unless indicated otherwise in a credit line to the material. If material is not included in the article's Creative Commons licence and your intended use is not permitted by statutory regulation or exceeds the permitted use, you will need to obtain permission directly from the copyright holder. To view a copy of this licence, visit http://creativecommons.org/licenses/by/4.0/.

(c) The Author(s) 2021 